Genetic polymorphism
may predict response
to medication in patients with alcoholism

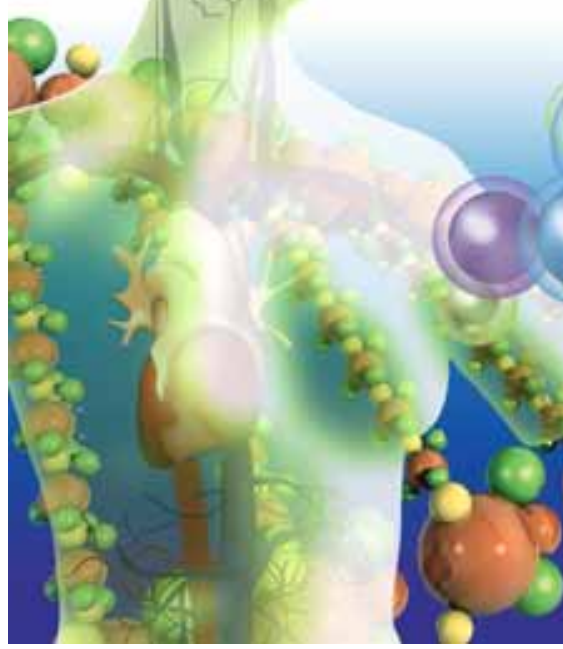

A new study shows that patients carrying a particular gene variant have a better response to the medication nal trexone compared with patients not carrying the variant. The patients involved were all participants in the N IH 's 2001-2004 Combined Pharmacotherapies and Behavioral Interventions for Alcohol D ependence (CO M BIN E) study.

The feeling of pleasure or well-being associated with imbibing alcohol is due to enhanced release of endogenous opioids, chemicals that increase these pleasurable feelings. $\mathrm{N}$ altrexone is an opioid antagonist and is used in the treatment of alcohol dependence. It exerts its action by binding to the same receptors as the endogenous opioids and blocking them, thereby aiding patients to remain abstinent. H owever, studies have shown a variation in response to the medication. Previously it has been suggested that the Asn40Asp variant, a functional polymorphism of the $\mu$-opioid receptor gene (OPRM 1), plays a role in predicting the response to naltrexone.

The aim of this study was to assess the response of patients with alcoholism, who were either heterozygous or homozygous for the Asp40 allele of OPRM 1, to naltrexone. Approximately 600 individuals (all participants of the COM BINE study) were included in the initial analysis, in which they weretreated for 16 weeks with either naltrexone hydrochloride or placebo.

The main variables measured to evaluate outcome were the percentage of days that an individual abstained from alcohol, the percentage of days when an individual drank heavily, and the rates of good clinical outcome. The latter was defined as complete abstinence or moderate drinking in conjunction with an absence of related problems. In addition, all patients also received medical management either on its own or in concert with combined behavioral intervention (CBI).

The scientists found that approximately $87 \%$ of Asp 40 carriers responded favorably to naltrexone treatment compared with approximately $49 \%$ of those who received a placebo. Approximately $55 \%$ of individuals without at least one copy of the Asp40 allele showed a good clinical outcome irrespective of whether they were treated with naltrexone or placebo.

Interestingly, the researchers did not observe any gene-medication interactions in the individuals who had received both medical management and CBI. This indicated that OPRM 1 genotyping may be of greatest use when the patient is treated with naltrexone without any additional intensive counseling.

'This study highlights the promise of truly personalized medic ine and could help to move trea tment of alc ohol dependence into the medical mainstrea m.'

"Analysis of the large CO M BINE patient population increases confidence that the OPRM 1 variant is in part responsible for positive responses to naltrexone. This study points to the promise of research on gene- medication interactions to refine treatment selection, improve clinical results and inform ongoing medications development," explained Ting-Kai $\mathrm{Li}$, director of the $\mathrm{N}$ ational Institute on Alcohol Abuse and Alcoholism (NIAAA).

"Research studies designed to ensure appropriate medication targeting are critical, especially as treatment for alcohol use disorders increasingly involves primary care physicians as well as specialists," commented $M$ ark $L$ Willenbring, the director of N IAAA's D ivision of Treatment and Recovery Research. "W ithout the ability to predict response for a specific patient, we must use trial-and-error to determine the correct medication - a process that may prolong illness and lead to more side effects. This study highlights the promise of truly personalized medicine and could help to move treatment of alcohol dependence into the medical mainstream."

Source: Anton RF, O roszi G, $O$ 'M alley $S$ et al.: An evaluation of $\mu$-opioid receptor (OPRM 1 ) as a predictor of naltrexone response in the treatment of alcohol dependence: results from the Combined Pharmacotherapies and Behavioral Interventions for Alcohol D ependence (CO M BIN E) study. Arch Gen Psychiatry 65(2), 135-144 (2008). 


\section{in brief...}

Genetic polymorphisms of TNF receptor superfamily $1 A$ and $1 B$ (TNFRSF1A and TNFRSF1B) affect responses to infliximab in Crohn's disease patients in Japan.

Matsukura H, Ikeda S, Yoshimura N, Takazoe M, Muramatsu M : Aliment Pharmacol. Ther. (2008) (Epub ahead of print).

Previous research has established the tumor necrosis factor $\alpha(T N F \alpha)$, an inflammatory cytokine, as one of the key mediators of the pathogenesis of Crohn's disease (CD). Infliximab, which is a chimeric monoclonal antibody of TNF $\alpha$, is used to treat $C D$, but the response to it varies. The aim of this study was to analyze the effect, if any, of polymorphisms in the genes encoding the TNF receptor superfamily $1 \mathrm{~A}$ and $1 \mathrm{~B}$ (TNFRSF1A and TNFRSF1B), the receptors that TNF $\alpha$ acts through, on the response to infliximab. A total of 80 patients, classed as either responder or nonresponder, were genotyped for SNPS of TNFRSF1A (rs767455 and rs4149570) and TNFRSF1B (rs1061622, rs1061624 and rs3397). W ith regard to TNFRSF1A, patients carrying the rs767455 allele showed a poorer response to treatment. The authors concluded that their results indicate a role for TNF receptor genotypes in the varied response to infliximab treatment in Japanese patients.

\section{HLA-B*5701 screening for hypersensitivity} to abacavir.

M allal S, Phillips E, Carosi G et al.: N. Engl. J. Med. 358(6), 568-579 (2008).

A strong association between the hypersensitivity reaction to abacavir and the HLA-B*5701 allele has been documented previously. The aim of this study was to evaluate the efficacy of prospective HLA-B* 5701 screening to prevent this adverse reaction. The trial consisted of over 1900 patients from 19 countries, all of whom were infected with HIV and had not been treated with abacavir. Patients were either screened for HLA-B*5701, with positives being excluded from abacavir treatment, or treated with abacavir as standard without any screening. The HLA-B*5701 allele was present in approximately 100 of the patients involved. After an observation period of 6 weeks, the researchers found that screening for the HLAB*5701 allele abolished the hypersensitivity reaction. A total of 93 patients were found to have a hypersensitivity reaction, the incidence of which was significantly reduced in the prospectivescreening group relative to the control group. The authors concluded that screening for the HLAB*5701 allele lowered the risk of an adverse hypersensitivity reaction to abacavir, and can play a key role in preventing a known toxic effect of a medication.

\section{Study finds novel mechanism for resistance acquired by some ovarian tumors to platinum-based chemotherapy}

Scientists at the Fred Hutchinson Cancer Research Center location have uncovered a novel mechanism underlying the resistance acquired by some recurrent ovarian tumors to the widely used platinum-based chemotherapy drugs like cisplatin and carboplatin. Although these new results are based on ovarian cancer sufferers carrying inherited mutations in the BRCA2 gene, it is possible that they could be extrapolated to BRCA1 as well and help explain the mechanism of cisplatin resistance in women with mutations in BRCA1.

"Because BRCA1 and BRCA2 have similar functions in terms of D N A repair, we may be able to generalizethesefindings for women with either mutation," explained Toshiyasu Taniguchi, senior author of the paper.

BRCA2 is involved in DN A repair, an ability that is disrupted by mutations, thereby leading to an increased risk of ovarian and breast cancer. $\mathrm{H}$ owever, at the same time these mutations also make the cells more sensitive to drugs like cisplatin, whose action is mediated via D N A damage. As a result, ovarian tumours respond very well to agents, such as cisplatin, but over time approximately $70-80 \%$ of patients acquire resistance to them. "T he majority of advancedstage ovarian-cancer patients die due to acquired resistance to platinumbased drugs. It is a serious problem," stated Taniguchi.

In this study, the scientists found that in some cases exposure to cisplatin resulted in the development of secondary mutations in the BRCA2 gene of the cancer cells. These mutations reinstated the $D N A$ repairing abilities of BRCA2, leading to the development of resistance to chemotherapeutic agents.

"Testing whether relapsed tumors havea secondary mutation of $B R C A 2$ outcome," commented Taniguchi.

"T his event is unlike any previous mechanism of resistance to chemotherapy identified in cancers," enthused Elizabeth Swisher, coauthor of the article. "By identifying the cause of chemotherapy resistance in these cancers, we may be able to better predict who will respond to different chemotherapy agents."

Source: Sakai W, Swisher EM , Karlan BY et al.: Secondary mutations as a mechanism of cisplatin resistance in BRCA2-mutated cancers. N ature (2008) (Epub ahead of print). may be important to predict clinical

\footnotetext{
About the Bulletin Board

The Bulletin Board highlights some of the most important events and launches in pharmacogenomics. The editorial team welcomes suggestions for timely, relevant items. If you have newsworthy information, please contact:

Shreeya Nanda, Commissioning Editor,

Pharmacogenomics, Future Medicine Ltd, Unitec House, 2 Albert Place,

London, N3 1QB, UK

s.nanda@futuremedicine.com

Tel.: +44 (0)20 83716090 Fax: +44 (0)20 83432313
} 


\section{Cystic fibrosis-associated bone complications may have a genetic basis}

\author{
$\mathrm{N}$ ew research at the $\mathrm{M}$ cG ill University \\ $\mathrm{H}$ ealth Centre Research Institute \\ indicates that a genetic factor may \\ underlie the bone problems that are often \\ associated with cystic fibrosis. This new \\ work could have implications in altering \\ therapeutic procedures. \\ Cystic fibrosis is a childhood-onset \\ hereditary disease primarily affecting the \\ respiratory and digestive systems. It is a \\ consequence of a mutation in the cystic \\ fibrosis transmembrane conductance \\ regulator (Cftr) gene. \\ It is generally believed that the cystic \\ fibrosis-associated bone frailty is \\ multifactorial, resulting from a \\ combination of mutation of the $\mathrm{Cftr}$ \\ gene, the pancreatic disease that \\ accompanies the disorder and the
}

\author{
steroid treatment given to help \\ with breathing. \\ Thisstudy used a mouse model in order \\ to analyze the bone phenotype of mice \\ lacking both copies of the $\mathrm{C}$ ftr gene in \\ relation to control mice, which were either \\ wild-type or heterozygous for the $\mathrm{Cftr}$ \\ gene. U sing a variety of histologic and \\ imaging analyses, the researchers found \\ that the C ftr-null mice were not only \\ smaller, but also had a significantly lower \\ bone mineral density and bone mass. This \\ difference was observed even though the \\ pancreatic deficiency normally seen in a \\ clinical situation was absent, as was the \\ steroid treatment, suggesting that the bone \\ phenotype associated with cystic fibrosis is \\ a consequence of the $\mathrm{Cftr}$ mutation and \\ not just a side effect of the medication.
}

This research paves theway for establishing a targeted treatment in mice, which could eventually be used to help patients.

The mechanism underlying the association between mutation in $\mathrm{Cftr}$ and bone metabolism is yet to be elucidated; however, studying these mice at various stages of development demonstrated that the bone structures of the $\mathrm{Cftr}$ null mice became more like the norm as they matured, indicating that the mutation only slows the growth of the bone and doesn't completely inhibit it. Further research is needed to confirm this hypothesis. Source: H aston CK, Li W, Li A, Lafleur M, $H$ enderson JE: Persistent osteopenia in adult cystic fibrosis transmembrane conductance regulator-deficient mice. Am. J. Respir. Crit. Care M ed. 177(3), 309-315 (2008).

\section{Gene plays a dual role in brain tumor development}

Glioblastoma is an aggressive and

highly malignant type of brain tumor, which is quite rare but almost always results in death. There are few treatment choices, and the ones that exist have remained more or less unchanged over the past years.

Previously, it has been shown that activation of the transcription factor STAT 3 leads to tumor development in a wide range of tumors; therefore, inhibiting this gene would be crucial to treating various tumors. H owever, contrary to previous work, a new study has discovered that STAT 3 doesn't always act as an oncogene, and it can actually act as a tumor suppressor in some cases, depending on the genetic profile of the tumor.

"T his discovery lays the foundation for a more tailored therapeutic intervention," commented Azad Bonni, senior author of the study. "And that's really important. You can't just go blindly treating people by inhibiting STAT 3."
M ost brain tumoursoccur in astrocytes, a type of brain cell that functions as a support cell. AsST AT 3 playsan important role in the development of astrocytes from neural stem cells, it may also play a part in glioblastoma development. It has been shown that the mutations in the EGFR and PTEN genes are associated with glioblastoma, and the researchers investigated how they impact upon the function of STAT 3 in astrocytes. Interestingly, they found that in the event of a mutation in EGFR, STAT 3 functions as an oncogene; however, when PTEN is mutated, STAT 3 acts as a tumor suppressor.

"The belief that STAT3 can only be an oncogene hasbeen a pretty entrenched dogma in the field, so we performed many, many experiments to make sure this was correct."

"EGFR, in its normal state, is a transmembrane receptor, usually performing its functions at the cell surface," explained Bonni. "H owever, when it's mutated, we find it in the cell's nucleus interacting with STAT 3 and turning it into an oncogene. STAT 3 itself is not mutated or damaged. It's the process of regulating STAT 3 that gets damaged."

"The belief that STAT 3 can only be an oncogene has been a pretty entrenched dogma in the field," concluded Bonni, "so we performed many, many experiments to make sure this was correct. It took some very persistent investigators in my lab to get the job done. As a result, we're convinced of our data."

Although glioblastomas are rare, STAT 3 is thought to be involved in tumorigenesis in prostate and breast cancers, and these findings could be translated to these other tumor types. Source: de la I glesia N, Konopka G, Puram SV et al.: Identification of a PTEN -regulated STAT 3 brain tumor suppressor pathway. Genes D ev. (2008) (Epub ahead of print). 